\title{
NONLINEAR NORMAL MODES AND THEIR APPLICATION IN STRUCTURAL DYNAMICS
}

\author{
CHRISTOPHE PIERRE, DONGYING JIANG, AND STEVEN SHAW
}

Received 12 February 2005; Revised 13 June 2005; Accepted 12 July 2005

Recent progress in the area of nonlinear modal analysis for structural systems is reported. Systematic methods are developed for generating minimally sized reduced-order models that accurately describe the vibrations of large-scale nonlinear engineering structures. The general approach makes use of nonlinear normal modes that are defined in terms of invariant manifolds in the phase space of the system model. An efficient Galerkin projection method is developed, which allows for the construction of nonlinear modes that are accurate out to large amplitudes of vibration. This approach is successfully extended to the generation of nonlinear modes for systems that are internally resonant and for systems subject to external excitation. The effectiveness of the Galerkin-based construction of the nonlinear normal modes is also demonstrated for a realistic model of a rotating beam.

Copyright (c) 2006 Christophe Pierre et al. This is an open access article distributed under the Creative Commons Attribution License, which permits unrestricted use, distribution, and reproduction in any medium, provided the original work is properly cited.

\section{The nonlinear normal mode}

In the design-analysis cycle of complex structural systems such as rotorcraft, aircraft, and ground vehicles, it is necessary to thoroughly understand their vibratory response. If the vibration of interest is restricted to small neighborhoods of the static equilibrium positions, then the assumption of a linear system can generally be made. The corresponding analysis procedure is then greatly simplified, through the use of modern tools such as Finite Element Analysis and Modal Analysis. In contrast, when the amplitudes of oscillation are large, beyond the scale of linearization, or when a system behaves inherently nonlinearly with respect to its equilibrium configuration, then nonlinear equations of motion must be used for the model.

In the area of nonlinear vibrations, much research work has been done for systems with a few degrees of freedom (DOF), such as lumped-mass models, and models obtained via a Galerkin discretization of the governing partial differential equations (PDE)

Hindawi Publishing Corporation

Mathematical Problems in Engineering

Volume 2006, Article ID 10847, Pages 1-15

DOI 10.1155/MPE/2006/10847 
2 Nonlinear normal modes and application in dynamics

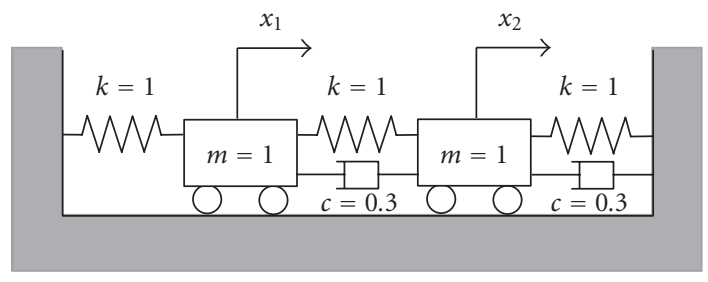

Figure 1.1. A two-degree-of-freedom linear mass-spring-dashpot system.

for continuous systems. These low-order models are useful for understanding general behavior. However, many of the methods developed are applicable only to relatively simple systems, and become unwieldy when used for systems modeled by complex PDEs or with a large number of DOFs.

In order to obtain accurate reduced-order models for nonlinear systems, "nonlinear modal analysis" has been proposed as an analog to its linear counterpart. The concept of nonlinear normal modes (NNM) was first developed by Rosenberg [9] for conservative systems with symmetric nonlinearities, essentially as an extension of the linear normal mode idea. A detailed review is provided by Vakakis [11], which summarizes much of the research in this area to date.

The research presented herein aims at the development and implementation of new model reduction methods for nonlinear structural systems, based on an invariant manifold approach proposed by Shaw and Pierre [10]. In order to illustrate the fundamental concept of the invariant manifold-based reduced-order modeling approach, a linear system is shown in Figure 1.1. The governing equation of motion is written in the state space as follows:

$$
\dot{z}=A z
$$

where the state variable $z=\left[\begin{array}{llll}x_{1} & y_{1} & x_{2} & y_{2}\end{array}\right]^{T}$, and velocities are $y_{i}=\dot{x}_{i}$. The determination of the state matrix $A$ is trivial and it is not given here. To construct the invariant manifold, the pair of state variables for the left mass is chosen as the master coordinates, $(u, v)=$ $\left(x_{1}, y_{1}\right)$. The remaining state variables, $\left(x_{2}, y_{2}\right)$, are denoted as the slave coordinates, and are expressed as

$$
x_{2}=X_{2}(u, v), \quad y_{2}=Y_{2}(u, v) .
$$

The invariant manifolds are determined by the constraint relationship in (1.2) and the equations of motion. Two such manifolds result, one for each mode. The resulting geometry of the invariant manifold for system (1.1) is shown in Figure 1.2. As can be seen, the manifold is planar for this linear system. Consequently, the invariant manifold approach reduces to the well-known linear mode results in the linearized case, although it offers a formulation that is entirely different from the traditional one [10]. 
Christophe Pierre et al. 3

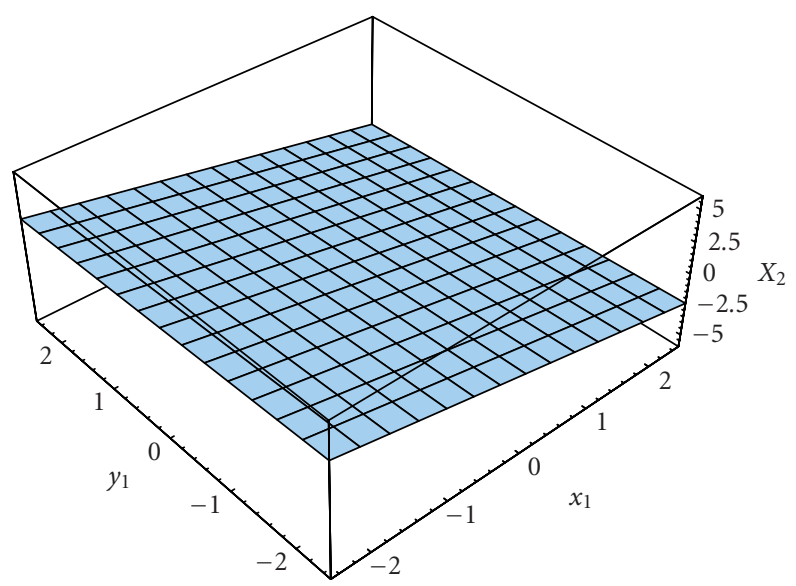

(a)

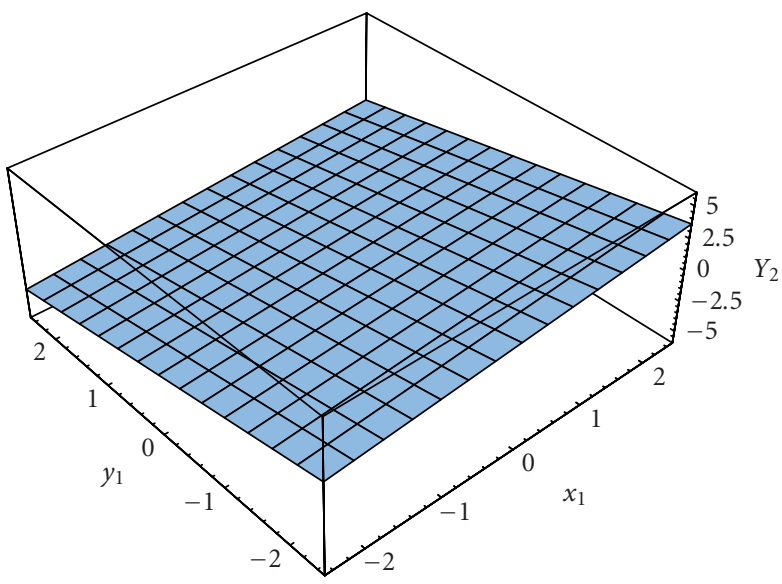

(b)

Figure 1.2. A modal invariant manifold for the linear two-DOF mass-spring-dashpot system: (a) $x_{2}=$ $X_{2}(u, v) ;(b) y_{2}=Y_{2}(u, v)$; where $(u, v)=\left(x_{1}, y_{1}\right)$.

The invariant manifold approach can be conveniently extended to nonlinear vibratory systems. For the two-DOF nonlinear system shown in Figure 1.3, the master coordinates are chosen as $(u, v)=\left(x_{1}, y_{1}\right)$, and the slave constraint relationships are defined in the same way as in (1.2). The invariant manifold can be approximated using the asymptotic method [10] and it is shown in Figure 2.1. As can be seen, the manifold for the nonlinear system is a curved surface.

The definition of an invariant manifold is related to its inherent property: invariance. The invariance of a manifold indicates that the motion initiated on the manifold remains 
4 Nonlinear normal modes and application in dynamics

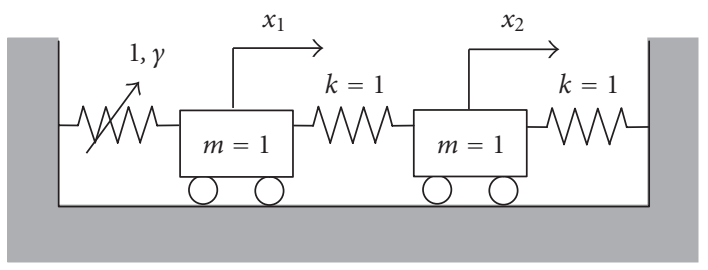

Figure 1.3. A two-degree-of-freedom nonlinear mass-spring system (a nonlinear spring with cubic nonlinearity is attached to mass one).

on that manifold for all time [10]. The nonlinear normal mode is defined on the invariant manifold as follows: "A motion that takes place on a two-dimensional invariant manifold in the system's phase space" [10]. This manifold passes through the stable equilibrium point of interest and, at that point, is tangent to a two-dimensional (linear mode) eigenspace of the linearized system about that equilibrium. On this manifold, the system behaves like a single-degree-of-freedom system.

The invariant manifold approach can be used to define and construct nonlinear normal modes of motion for a wide class of nonlinear vibratory systems $[2,6,10,11]$. The following sections give an overview of the construction methods and the applications of nonlinear normal modes.

\section{The construction of the nonlinear normal mode: asymptotic approach versus Galerkin method}

A $n$-DOF vibratory system can be expressed in the following first-order form:

$$
\dot{x}=y, \quad \dot{y}=f(x, y), \quad x, y \in \mathfrak{R}^{N},
$$

where the $n$-dimensional vectors $x$ and $y$ are the system linear modal displacements and linear modal velocities, respectively. The use of linear modal coordinates in (2.1) offers a convenient standard form from which to construct the invariant manifold-based nonlinear normal mode [7].

The asymptotic approach is outlined here for the construction of the invariant manifold. First, multiple pairs of linear modal coordinates (displacement-velocity) are chosen as the master coordinates, the "seeds" of the invariant manifold:

$$
\left(u_{k}, v_{k}\right), \quad k \in S_{M}
$$

where $\left(u_{k}, v_{k}\right)$ denotes the $k$ th linear modal displacement-velocity pair, and $S_{M}$ denotes the set of the master coordinate indices. Then, all the remaining degrees of freedom in (2.1) are constrained to the master coordinates and approximated in series form as 


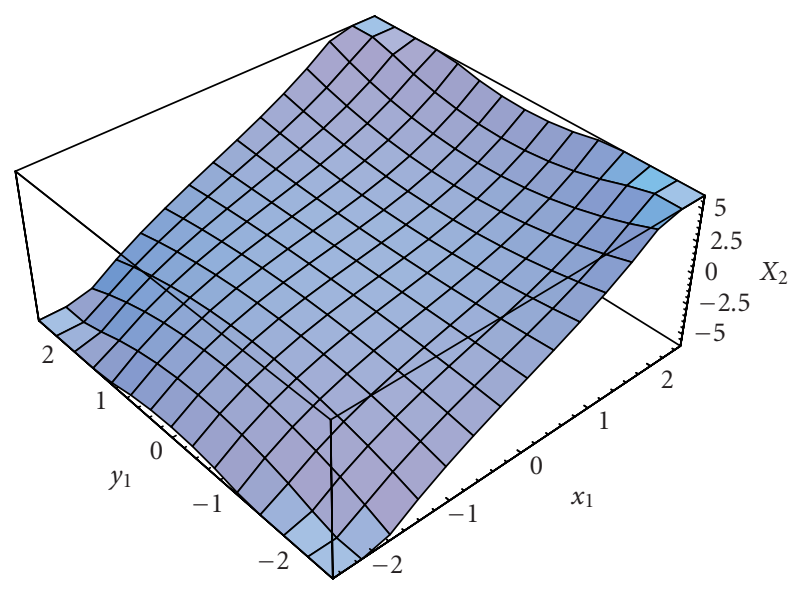

(a)

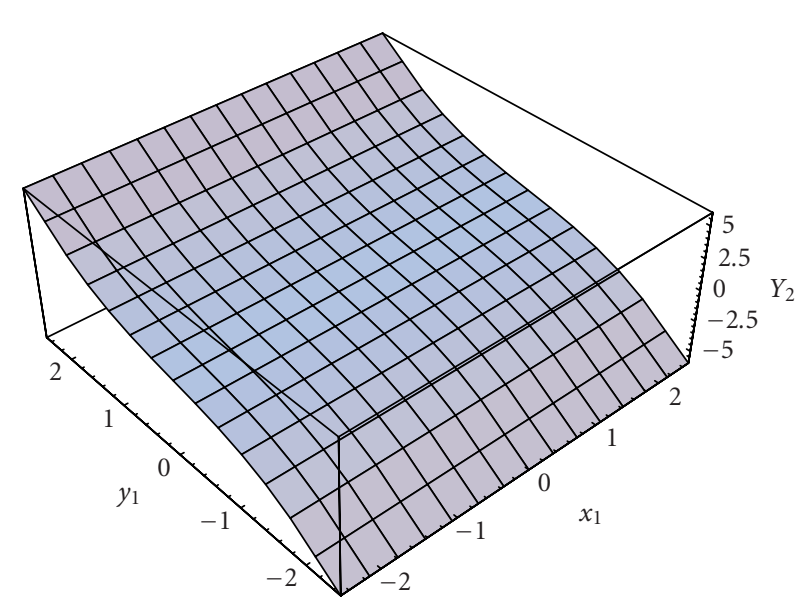

(b)

Figure 2.1. A modal invariant manifold for the nonlinear two-DOF mass-spring system: (a) $x_{2}=$ $X_{2}(u, v)$; (b) $y_{2}=Y_{2}(u, v)$; where $(u, v)=\left(x_{1}, y_{1}\right)$.

follows:

$$
\begin{aligned}
x_{j}\left(u_{M}, v_{M}\right)= & \sum_{k \in S_{M}} a_{1, j}^{k} u_{k}+a_{2, j}^{k} v_{k} \\
& +\sum_{k \in S_{M}} \sum_{l \in S_{M}} a_{3, j}^{k, l} u_{k} u_{l}+a_{4, j}^{k, l} u_{k} v_{l}+a_{5, j}^{k, l} v_{k} v_{l}+\cdots O(3), \\
y_{j}\left(u_{M}, v_{M}\right)= & \sum_{k \in S_{M}} b_{1, j}^{k} u_{k}+b_{2, j}^{k} v_{k} \\
& +\sum_{k \in S_{M}} \sum_{l \in S_{M}} b_{3, j}^{k, l} u_{k} u_{l}+b_{4, j}^{k, l} u_{k} v_{l}+b_{5, j}^{k, l} v_{k} v_{l}+\cdots O(3), \quad \text { for } j \notin S_{M} .
\end{aligned}
$$


In (2.3), the constraint relationships for the slave coordinates are approximated using asymptotic expansions, and the coefficients, the $a$ 's and $b$ 's, need to be determined. The solution of these coefficients has been studied by Pesheck et al. [8]. The detailed derivation is not repeated here, since the primary focus is on the more accurate Galerkin construction method.

The asymptotic approach has some inherent deficiencies: the constructed invariant manifold is limited to the domain near the equilibrium position, and this domain cannot be determined a priori; the accuracy of the manifold is constrained to the order of the polynomial used in the expansion; and the approach cannot be extended to systems with complex nonlinearities, especially for systems with nonsmooth nonlinearities such as gaps. In order to solve for the invariant manifold accurately, a numerical Galerkin approach has been proposed [7].

For the nonlinear system defined in (2.1), the multiple pairs of master coordinates, $\left(u_{k}, v_{k}\right)$ in $(2.2)$, are transformed to polar coordinate form, as follows:

$$
\begin{gathered}
u_{k}=a_{k} \cos \phi_{k}, \\
v_{k}=-a_{k} \omega_{k} \sin \phi_{k}, \quad k \in S_{M},
\end{gathered}
$$

where $\omega_{k}$ is the $k$ th linear modal frequency, and the multiple amplitude-phase pairs, $\left(a_{k}, \phi_{k}\right)$, are the new master coordinates. The slave coordinates are constrained to the new master coordinates as follows:

$$
x_{i}=P_{i}\left(a_{k}, \phi_{k}\right), \quad y_{i}=Q_{i}\left(a_{k}, \phi_{k}\right), \quad i \notin S_{M},
$$

where $P_{i}$ and $Q_{i}$ are the slave constraint functions. The governing PDEs for the invariant manifold are found [5] to be

$$
\begin{gathered}
Q_{i}=\sum_{k \in S_{M}}\left[\frac{\partial P_{i}}{\partial a_{k}}\left(-\frac{f_{k}}{\omega_{k}} \sin \phi_{k}\right)+\frac{\partial P_{i}}{\partial \phi_{k}}\left(\omega_{k}-\frac{f_{k}}{\omega_{k} a_{k}} \cos \phi_{k}\right)\right], \\
\sum_{k \in S_{M}}\left[\frac{\partial Q_{i}}{\partial a_{k}}\left(-\frac{f_{k}}{\omega_{k}} \sin \phi_{k}\right)+\frac{\partial Q_{i}}{\partial \phi_{k}}\left(\omega_{k}-\frac{f_{k}}{\omega_{k} a_{k}} \cos \phi_{k}\right)\right]=-\omega_{i}^{2} P_{i}+f_{i},
\end{gathered}
$$

where $f_{i}$ denotes the nonlinear force on the $i$ th linear modal coordinate.

The invariant manifold is determined by the slave constraint functions, $P_{i}$ 's and $Q_{i}$ 's in (2.6), which must be solved for numerically. The system dynamics on the invariant manifold are then governed by the master coordinates only. Hence, the original nonlinear system is reduced to the master coordinates. The detailed derivation of the partial differential equations governing the invariant manifold can be found in $[5,7]$, and is not repeated here.

The Galerkin method has the following advantages over the asymptotic approach in the construction of invariant manifolds: the range of validity of the manifold can be predetermined by setting the amplitude range of the master coordinates; the accuracy of the manifold can be controlled by the expansion functions in the master coordinates, the $a$ 's and $\phi$ 's; and the Galerkin formulation is applicable to systems with complex 


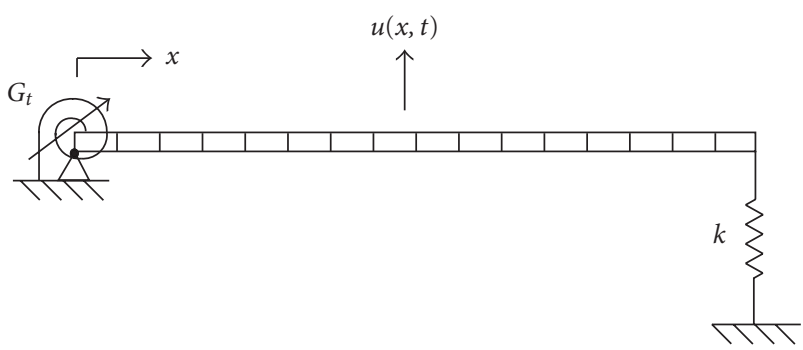

Figure 2.2. A finite element beam with nonlinear torsional spring at the left end: length of the beam $L=1 \mathrm{~m}$, density $\rho=7860 \mathrm{~kg} / \mathrm{m}^{3}$, beam bending stiffness $E I=10^{4} \mathrm{~N} \cdot \mathrm{m}^{2}$, linear spring stiffness $k=10^{8} \mathrm{~N} / \mathrm{m}$, nonlinear torsional force $G_{t}=5000 u_{x_{x}}(0, t)^{2}+20000 u_{x_{x}}(0, t)^{3} \mathrm{~N}$ (where $u_{,_{x}}$ denotes the partial derivative of $u(x, t)$ with respect to $x)$ [7].

nonlinearities [3] and nonlinear systems with external forcing [4]. Consequently, the Galerkin method seamlessly interfaces with finite element models of engineering structures, and it allows the user to specify the vibration amplitude range and the accuracy of the model over that range.

To illustrate the difference between the invariant manifolds constructed by the asymptotic approach and the Galerkin method, the finite element beam model shown in Figure 2.2 is considered [7]. The original beam model has 200 finite elements, and for illustrative purposes the system is truncated to three linear modal coordinates. The second nonlinear normal mode is examined. Specifically, the master coordinates are chosen as $\left(x_{2}, y_{2}\right)$ in the asymptotic approach and as $\left(a_{2}, \phi_{2}\right)$ in the Galerkin method. The slave constraint for the first linear modal displacement, $x_{1}$, is shown in Figure 2.3. As can be seen, the manifold obtained from the asymptotic approach deviates dramatically from the (more accurate) manifold obtained by the Galerkin method as the master coordinates enter the strongly nonlinear region. For this three-DOF beam model, it should be noted that there are four slave constraints, $x_{1}, y_{1}, x_{3}$, and $y_{3}$, which represent the full invariant manifold for the second nonlinear normal mode.

\section{The interaction between nonlinear normal modes: internal resonance}

The nonlinear normal mode construction procedure presented in Section 2 can be utilized to examine the nonlinear dynamics for the multiple modes involved in an internal resonance. The rotating rotorcraft blade shown in Figure 3.1 is used to illustrate these ideas; the blade model includes nonlinear bending-axial coupling and large-amplitude motions $[1,5]$. The weak formulation for the equation of motion of the blade is found [1] to be

$$
\begin{aligned}
\int_{t_{1}}^{t_{2}} \int_{0}^{L}\{ & {\left[-m \ddot{w}-E I w,_{x x x x}\right] \delta w-\left[E A\left(u,_{x}+\frac{1}{2}\left(w,_{x}\right)^{2}\right) w,_{x}\right] \delta w,_{x} } \\
+ & {\left[-m \ddot{u}+m \Omega^{2}(x+h+u)+E A u,_{x x}\right] \delta u } \\
& \left.-\left[E A \frac{1}{2}\left(w, x_{x}\right)^{2}\right] \delta u, x_{x}\right\} d x d t=0, \quad \forall t_{1}<t<t_{2},
\end{aligned}
$$


8 Nonlinear normal modes and application in dynamics

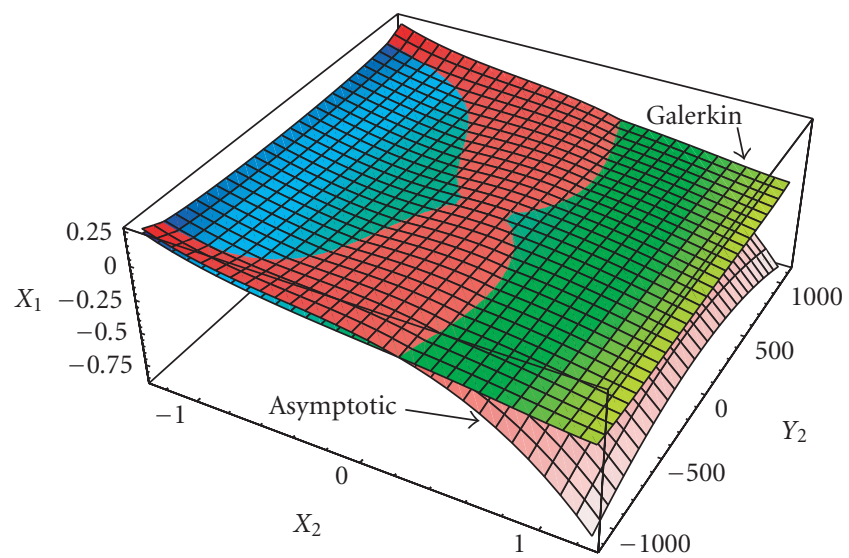

(a)

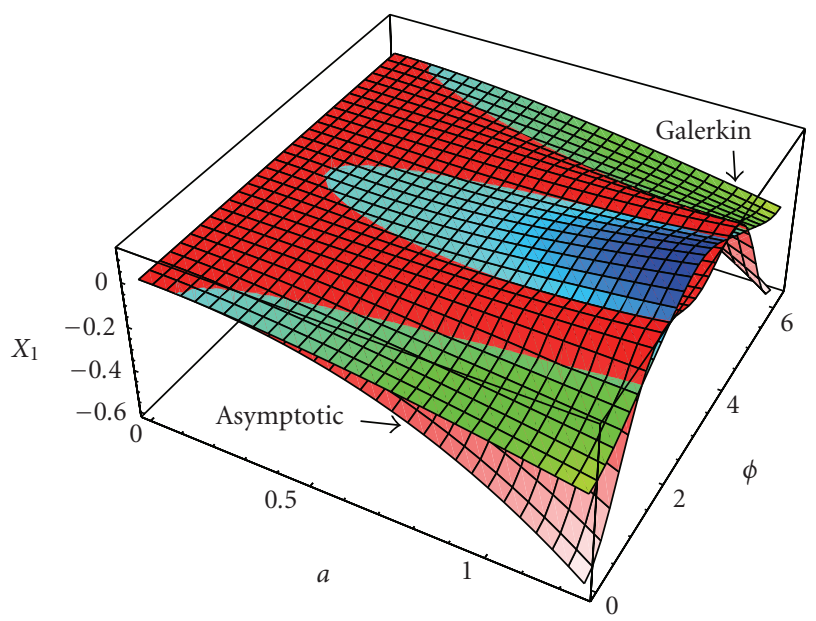

(b)

Figure 2.3. The asymptotic and Galerkin manifolds for the first linear modal coordinate contribution to the second nonlinear normal mode: (a) $x_{1}$ in terms of master coordinates $\left(x_{2}, y_{2}\right)$; (b) $x_{1}$ in terms of master coordinates $(a, \phi)[7]$.

where $w(x, t)$ and $u(x, t)$ are the transverse and axial displacement respectively; ()${ }_{x}$ denotes a partial derivative with respective to the spatial variable $x$, and $(\cdot)$ represents a time derivative; $\delta()$ denotes the variation of a quantity.

For the blade in Figure 3.1(a), a nonlinear finite element model has been developed that includes flapping motion and axial stretching [1]. In order to achieve adequate accuracy, 182 finite elements are needed. A linear modal analysis is carried out to construct the reference model, and 18 linear modes (9 flapping modes and 9 axial stretching modes) are retained in order to achieve sufficient modal convergence. Moreover, the blade model 


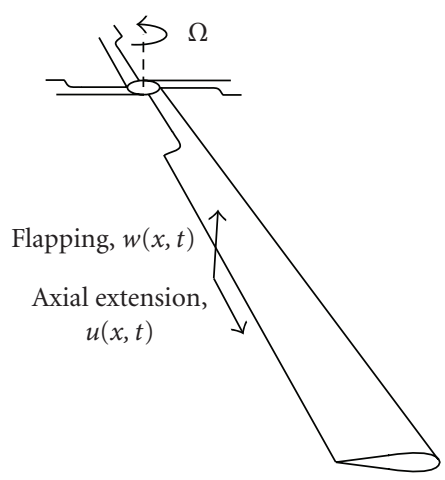

(a)



(b)

Figure 3.1. Schematic of the rotating blade model: (a) a blade with coupling between flapping and axial extension motions; (b) finite element representation of a rotating beam with constant angular velocity, $\Omega$. The beam has the following material and geometrical parameters: Young's modulus $E$, cross-section area $A$, second moment of cross-section area $I$, length $L$, and mass per unit length $m$. The hub radius is $h[1]$.

is tuned with an internal resonance between the first and the second flapping modes [5]: the modal frequency of the second flapping mode is approximately three times the first flapping modal frequency, $\omega_{2} \approx 3 \omega_{1}$.

By selecting the linear modal coordinates for the first and the second flapping modes as the master coordinates, $\left(a_{1}, \phi_{1}\right)$ and $\left(a_{2}, \phi_{2}\right)$, all the remaining degrees-of-freedom are slave coordinates, and the corresponding two-mode invariant manifold can be constructed using the Galerkin method [5]. A cross-sectional view of the invariant manifold (mode shape) for the nonlinear two-mode model is shown in Figure 3.2(a), where $a_{1}$ and $a_{2}$ denote the amplitude of the first and the second flapping linear modes, respectively, and $x_{3}$ denotes the linear modal displacement of the third flapping linear mode in terms of the amplitudes $a_{1}$ and $a_{2}$. There is a total of 16 similar cross-sectional views of the manifold, corresponding to all the slave constraint relationships that are constructed.

Once the nonlinear normal mode is constructed, the nonlinear dynamics on the invariant manifold are reduced to the master coordinates. The nonlinear mode-based reduced-order model is described by two second-order equations. As a result, the system motion can be very efficiently simulated, as shown in Figure 3.2(b). The nonlinear mode simulation matches the reference result very well. Note that the reference solution 
requires the direct time simulation of 18 coupled second-order differential equationsan expensive calculation. The result for the asymptotic-based reduced-order model is also shown: the asymptotic result is only valid in the weakly nonlinear domain, and it leads to qualitatively wrong results in the strongly nonlinear region. In these simulations, a peak-to-peak tip deflection of nearly 0.4 meters is obtained for this 9 -meter-long blade.

\section{The nonlinear normal mode for systems with harmonic excitation}

The invariant manifold-based nonlinear modal analysis approach developed in Section 2 is extended to systems that are subjected to periodic forces [4], thereby providing a useful tool for attacking the important problem of obtaining the frequency response of nonlinear structures.

In order to incorporate the external excitation into the vibratory system described by (2.1), the system's governing equation is expressed in the following augmented form:

$$
\begin{gathered}
\dot{x}=y, \quad \dot{y}=f\left(x, y, \phi_{f}\right), \quad x, y \in \mathfrak{R}^{N}, \\
\dot{\phi}_{f}=\omega_{f},
\end{gathered}
$$

where $\phi_{f}$ is the additional state phase variable for the external excitation, and $\omega_{f}$ is the frequency of harmonic excitation. To construct the invariant manifold, the master coordinates are chosen as the linear modal coordinates in the polar form, $\left(a_{k}, \phi_{k}\right)$ for $k \in S_{M}$, and the excitation state variable, $\phi_{f}$. The slave constraints are then expressed in the space spanned by the master coordinates as follows:

$$
x_{i}=P_{i}\left(a_{k}, \phi_{k}, \phi_{f}\right), \quad y_{i}=Q_{i}\left(a_{k}, \phi_{k}, \phi_{f}\right), \quad i \notin S_{M} .
$$

The derivation of the PDE's governing the invariant manifold and the Galerkin-based construction method for the nonlinear normal mode under external forcing are detailed in reference [4]. Once the nonlinear mode is constructed, the system response on the manifold can be reduced to the master coordinates, which include the system state variables and the forcing state variable.

An example beam system is shown in Figure 4.1 with harmonic external forcing. The equation of motion governing the beam deflection, $u(x, t)$, is given [4] in weak form as follows:

$$
\begin{aligned}
& \int_{t_{1}}^{t_{2}}\left\{\int_{0}^{L}\left(-\rho A \ddot{u} \delta u-E I u_{, x x} \delta u_{, x x}\right) d x-k u(L, t) \delta u(L, t)\right. \\
& \left.\quad-\gamma_{t} \delta u,_{x}(0, t)+f_{0} \cos \omega_{f} t \delta u(L, t)\right\} d t=0, \quad \forall t_{1}<t<t_{2} .
\end{aligned}
$$

For simplicity, the finite element beam model is truncated to 12 linear modes for the investigation of the primary resonance near the first linear modal frequency, $\omega_{1}=$ $222.4 \mathrm{rad} / \mathrm{s}$. The first linear modal coordinates are chosen as the master coordinates, $\left(a_{1}, \phi_{1}\right)$. The invariant manifold is constructed corresponding to the excitation frequency 


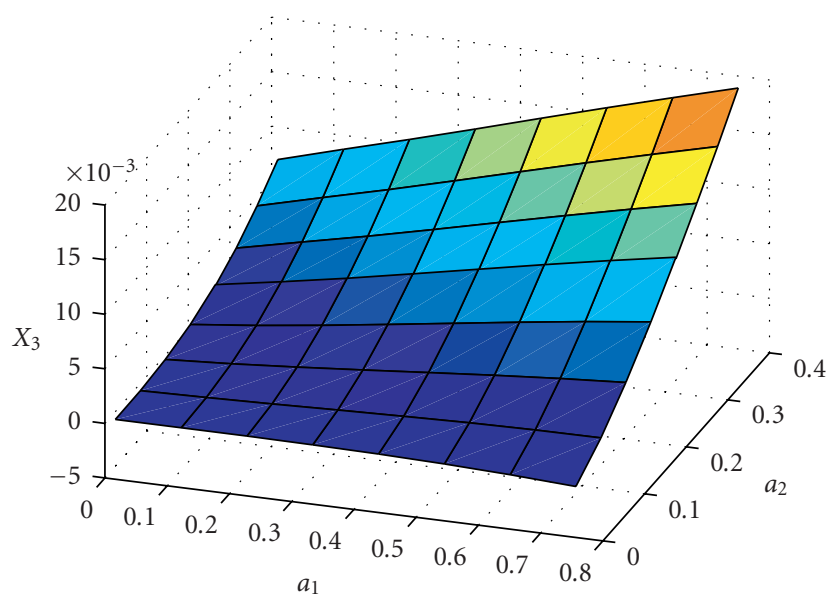

(a)

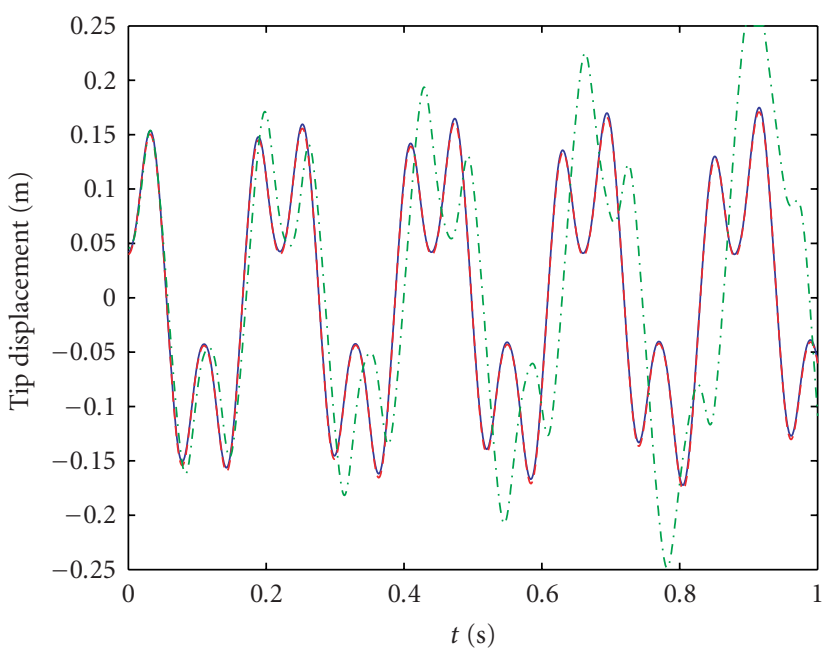

(b)

Figure 3.2. The invariant manifold and the time response on the manifold: (a) a cross-sectional view of the invariant (modal) manifold for the four-dimensional nonlinear normal mode; $X_{3}\left(a_{1}, a_{2}\right)$ at $\phi_{1}=\phi_{2}=0$; (b) time response for the blade tip displacement in the constructed four-dimensional nonlinear mode: the blue solid line refers to the 18-DOF reference model solution, the red dashed line represents the two-DOF reduced-order model, and the green dashed line denotes the result from the asymptotic-based reduced-order model [5].

$\omega_{f}[4]$. Once the nonlinear normal mode is constructed, the system dynamics near the first primary resonance can be investigated in the reduced-order model governing the master coordinates only. The steady-state response and the transient responses are shown in Figures 4.2-4.4. 
12 Nonlinear normal modes and application in dynamics

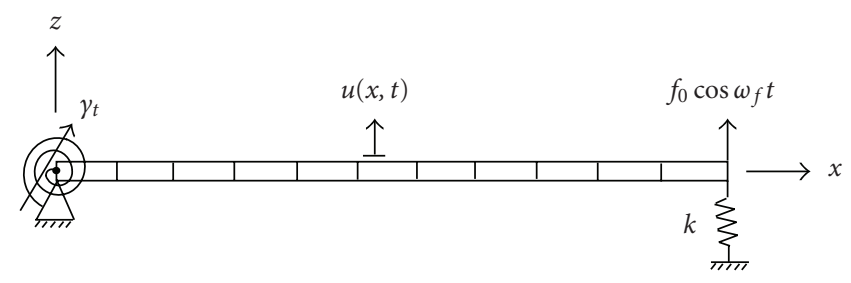

Figure 4.1. An Euler-Bernoulli beam with a nonlinear torsional spring, $\gamma_{t}$, at the left end, and a harmonic external excitation at the right end. The beam has the same material and geometrical properties as the beam in Figure 2.2. The amplitude of the force, $f_{0}$, is 3,000,000 N. The excitation frequency, $\omega_{f}$, is swept from $220 \mathrm{rad} / \mathrm{s}$ to $260 \mathrm{rad} / \mathrm{s}$ for the investigation of the first primary resonance [4].

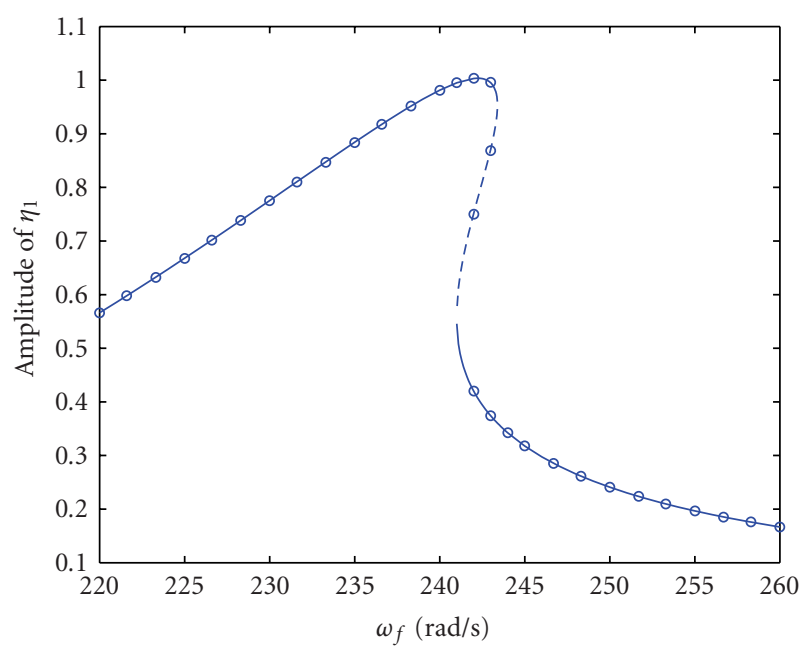

Figure 4.2. The amplitude of the steady-state response of the first linear modal coordinate (master coordinate), $\eta_{1}(\mathrm{t})$, versus the excitation frequency, $\omega_{f}$. The solid line refers to the stable steady-state response obtained from the original system model; the dashed line refers to the unstable steady-state response from the original system model; and the circle refers to the steady-state response from the Galerkin-based reduced-order model [4].

The steady-state response of master coordinate $\eta_{1}$ is shown in Figure 4.2. As can be seen, both the stable branches and the unstable branch of the response are accurately captured by the simulation based on the nonlinear mode-based reduced-order model. Note that at the excitation frequency $\omega_{f}=242 \mathrm{rad} / \mathrm{s}$, the amplitude of $\eta_{1}$ reaches its peak at 1.0 , which is physically equivalent to a $0.3 \mathrm{~m}$ displacement near the middle point of the one-meter-long beam. The example clearly demonstrates the capability of accurately capturing the forced response over a strongly nonlinear amplitude range.

The transient response at the excitation frequency, $\omega_{f}=242 \mathrm{rad} / \mathrm{s}$, is also shown in Figures 4.3 and 4.4 for two different initial conditions, each leading to a different steadystate response. As can be seen, the response from the reduced-order model matches very 


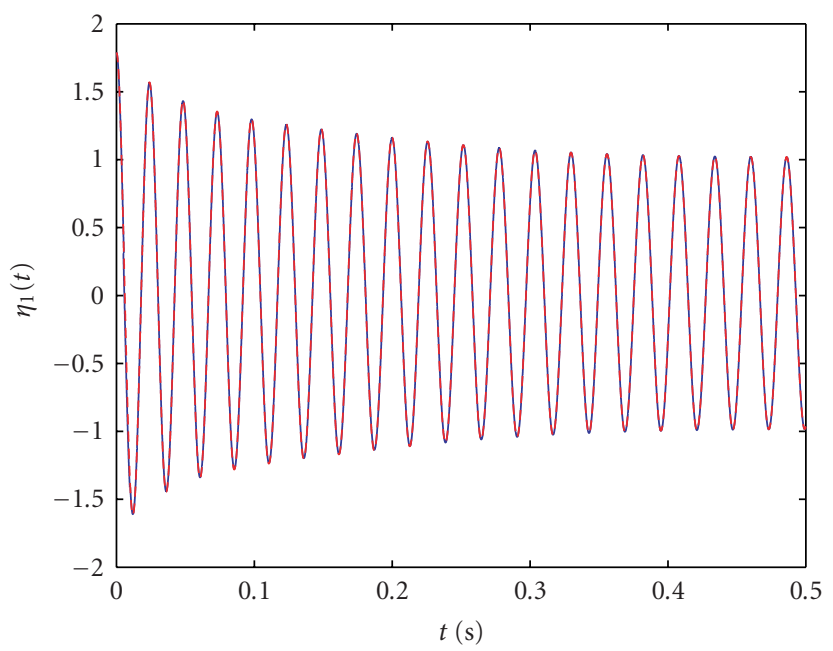

(a)

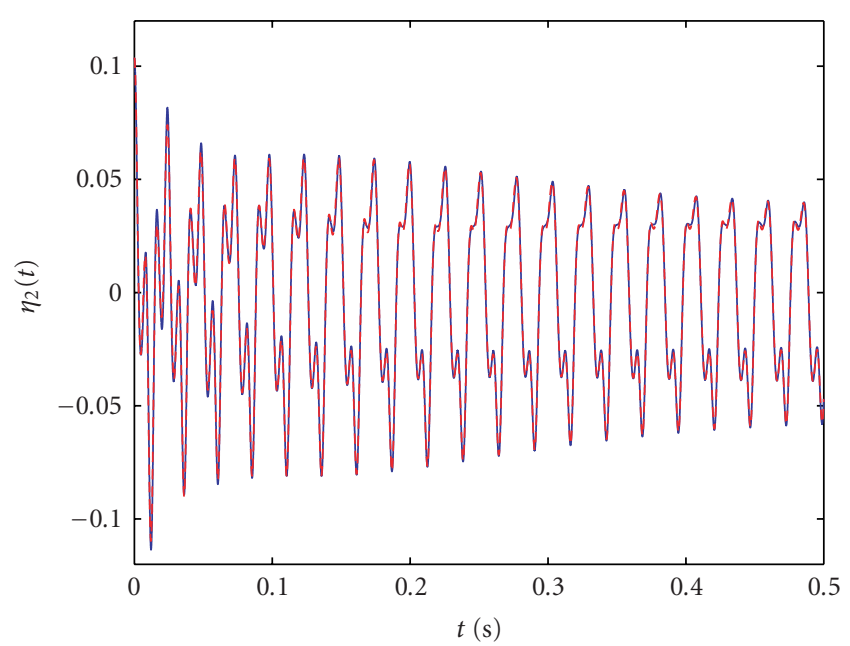

(b)

Figure 4.3. Comparison of the transient-forced response of the modal coordinates, $\eta_{1}(t)$ and $\eta_{2}(t)$, with initial conditions $a(0)=1.79, \dot{a}(0)=0.0$. the solid line refers to the response obtained from the original 12-DOF system model; the dashed line refers to the response from the reduced-order model $[4]$.

closely that from the original system for a range of initial conditions. This demonstrates that the dynamics near the primary resonance can be very accurately captured by the invariant manifold approach. Similar results can be obtained for other resonances, by choosing master modes accordingly. 
14 Nonlinear normal modes and application in dynamics

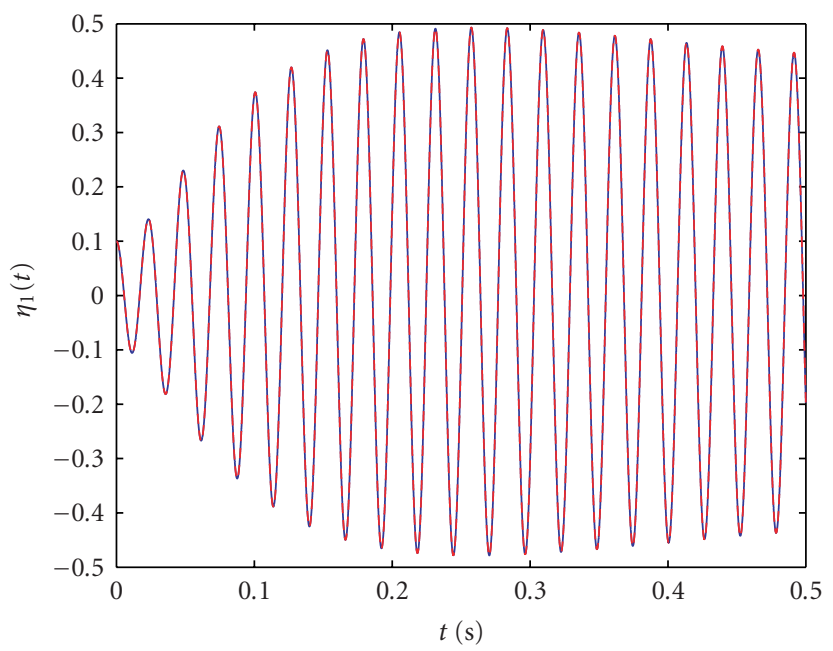

(a)

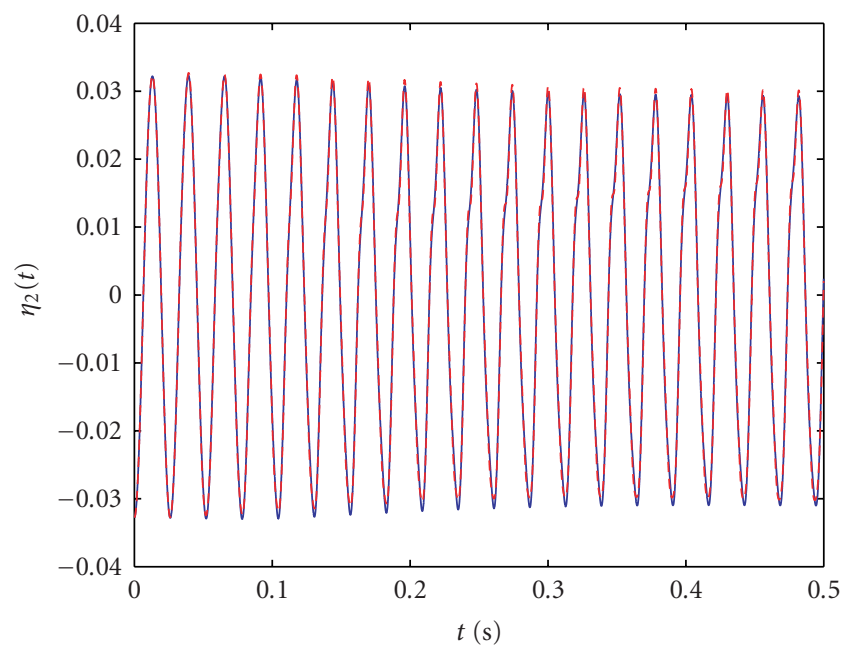

(b)

Figure 4.4. Comparison of the transient-forced response of the modal coordinates, $\eta_{1}(t)$ and $\eta_{2}(t)$, with initial conditions $a(0)=0.1$ and $\dot{a}(0)=0.0$. The solid line refers to the response obtained from the original 12-DOF system model; the dashed line refers to the response from the reduced-order model [4].

\section{Conclusions}

The essence of nonlinear modal analysis is to generate a minimal reduced-order model that accurately captures the dynamics of the original system. An effective normal modebased model order reduction methodology must satisfy two basic criteria. First, the 
method must be systematic, which means it can deal with various kinds of nonlinear forces and handle various structural models, such as finite element models. Second, the construction of the nonlinear normal modes must be numerically efficient, in the sense that the solution can be obtained from a computer code in a reasonable time, regardless of the size of the actual nonlinear system. The results obtained in this paper demonstrate that the invariant manifold-based approach is general enough to account for various types of nonlinear forces, including conventional quadratic and cubic nonlinear forces, and complex intrinsic nonlinear effects such as those in the model of a rotating rotorcraft blade. Also, the Galerkin-based solution technique developed has been shown to allow for the efficient generation of the invariant manifolds, for large nonlinear systems and up to large amplitudes. Therefore, this approach allows for the practical application of the invariant manifold formulation for a wide variety of engineering structures.

\section{References}

[1] P. Apiwattanalunggarn, S. W. Shaw, C. Pierre, and D. Jiang, Finite-element-based nonlinear modal reduction of a rotating beam with large-amplitude motion, Journal of Vibration and Control 9 (2003), no. 3-4, 235-263.

[2] L. Jézéquel and C. H. Lamarque, Analysis of non-linear dynamical systems by the normal form theory, Journal of Sound and Vibration 149 (1991), no. 3, 429-459.

[3] D. Jiang, C. Pierre, and S. W. Shaw, Large-amplitude non-linear normal modes of piecewise linear systems, Journal of Sound and Vibration 272 (2004), no. 3-5, 869-891.

[4] , Nonlinear normal modes for vibratory systems under harmonic excitation, Journal of Sound Vibration 288 (2005), no. 4-5, 791-812.

[5] _ The construction of nonlinear normal modes for systems with internal resonance: application to rotating beams, International Journal of Non-Linear Mechanics 40 (2005), no. 5, 729-746.

[6] H. Nayfeh and S. A. Nayfeh, On nonlinear modes of continuous systems, ASME Journal of Vibration and Acoustics 116 (1994), no. 1, 129-136.

[7] E. Pesheck, C. Pierre, and S. W. Shaw, A new Galerkin-based approach for accurate non-linear normal modes through invariant manifolds, Journal of Sound and Vibration 249 (2002), no. 5, 971-993.

[8] _ Model reduction of a nonlinear rotating beam through nonlinear normal modes, ASME Journal of Vibration and Acoustics 124 (2002), no. 2, 229-236.

[9] R. M. Rosenberg, The normal modes of nonlinear n-degree-of-freedom systems, Transactions of the ASME. Journal of Applied Mechanics 29 (1962), 7-14.

[10] S. W. Shaw and C. Pierre, Normal modes for nonlinear vibratory systems, Journal of Sound and Vibration 164 (1993), no. 1, 85-124.

[11] A. F. Vakakis, Non-linear normal modes and their applications in vibration theory: an overview, Mechanical Systems and Signal Processing 11 (1997), no. 1, 3-22.

Christophe Pierre: Department of Mechanical Engineering, University of Michigan,

Ann Arbor, MI 48109, USA

E-mail address: pierre@umich.edu

Dongying Jiang: MKP Structural Design Associates, Inc., Ann Arbor, MI 48104, USA

E-mail address: jiang@mkpsd.com

Steven Shaw: Department of Mechanical Engineering, Michigan State University, East Lansing, MI 4882, USA

E-mail address: shawsw@msu.edu 


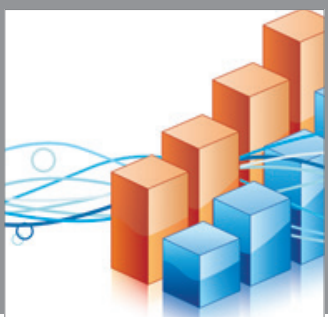

Advances in

Operations Research

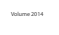

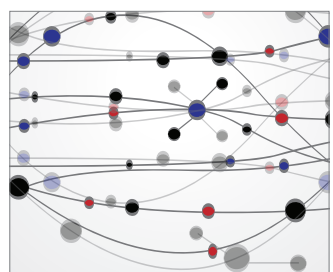

\section{The Scientific} World Journal
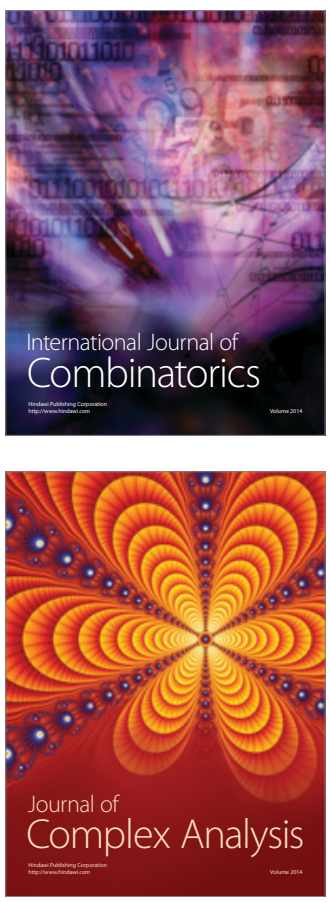

International Journal of

Mathematics and

Mathematical

Sciences
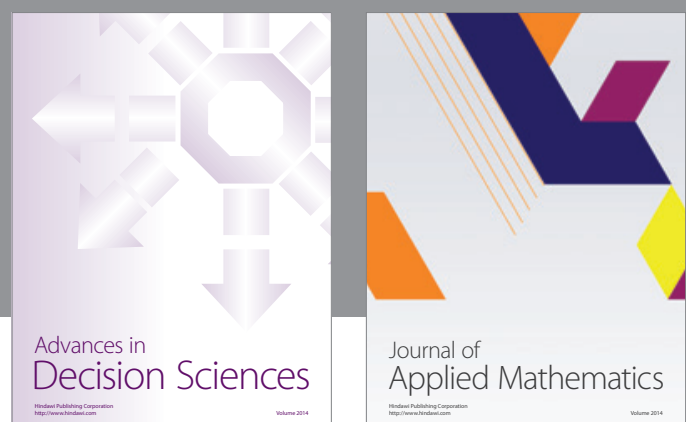

Journal of

Applied Mathematics


Submit your manuscripts at http://www.hindawi.com
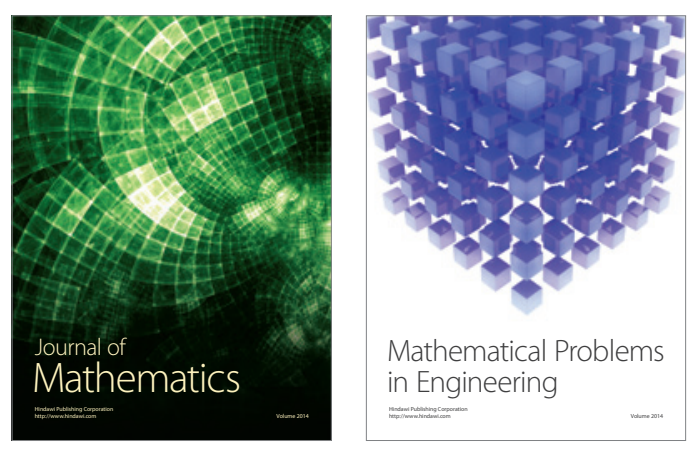

Mathematical Problems in Engineering
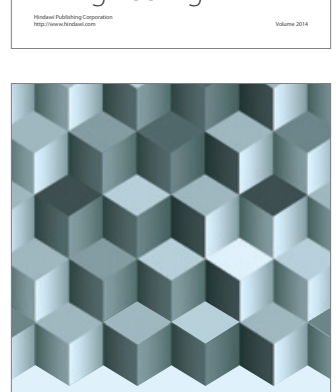

Journal of

Function Spaces
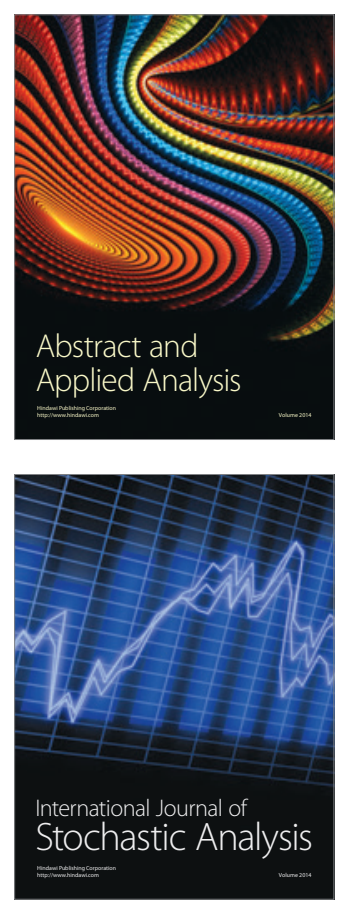

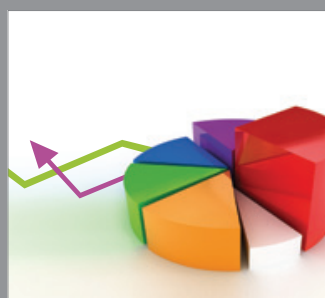

ournal of

Probability and Statistics

Promensencen
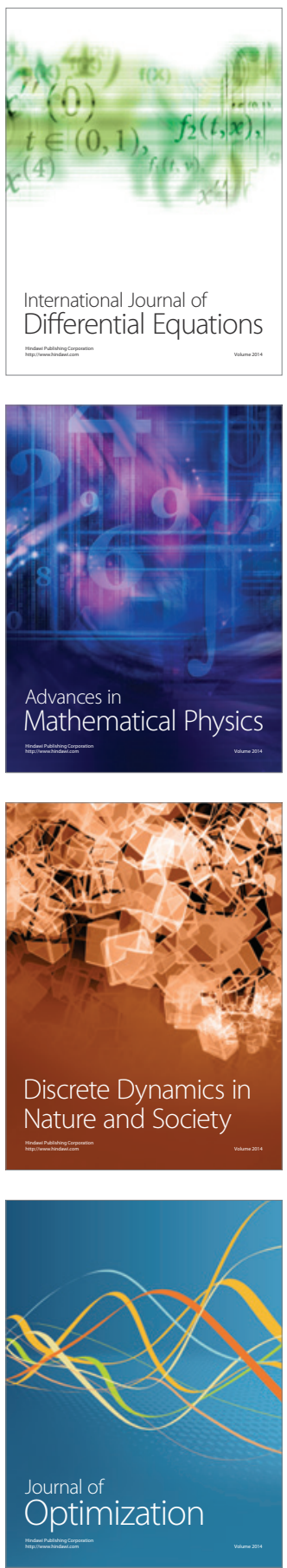